Revue

Revue de l'histoire des religions

de Ihistoire

des religions

$3 \mid 2016$

Varia

\title{
Les sciences religieuses au défi du comparatisme : notes critiques
}

Religious Science and the Challenge of the Comparative Approach: Critical Notes

\section{Viviane Comerro de Prémare et Alain Rauwel}

\section{OpenEdition}

\section{Journals}

Édition électronique

URL : http://journals.openedition.org/rhr/8561

DOI : 10.4000/rhr.8561

ISSN : 2105-2573

\section{Éditeur}

Armand Colin

\section{Édition imprimée}

Date de publication : 1 septembre 2016

Pagination : 291-303

ISBN : 978-2-200-93061-5

ISSN : 0035-1423

Référence électronique

Viviane Comerro de Prémare et Alain Rauwel, «Les sciences religieuses au défi du comparatisme : notes critiques », Revue de l'histoire des religions [En ligne], 3 | 2016, mis en ligne le 01 septembre 2019, consulté le 07 septembre 2020. URL : http://journals.openedition.org/rhr/8561 ; DOI : https://doi.org/ $10.4000 /$ rhr.8561 
VIVIANE COMERRO DE PRÉMARE

Institut national des langues

et civilisations orientales, Paris
ALAIN RAUWEL

Université de Bourgogne

\title{
Les sciences religieuses au défi du comparatisme Notes critiques*
}

\begin{abstract}
Plusieurs ouvrages sont venus récemment proposer au public francophone des instruments pratiques en vue d'une compréhension globale du phénomène monothéiste. Deux d'entre eux sont examinés ici. Il apparaît que la pierre de touche de telles entreprises est la démarche comparatiste. La simple juxtaposition de notices informatives ne saurait en tenir lieu, mais la rédaction de synthèses plus ambitieuses court aussi le risque de s'appuyer sur une connaissance inégale des univers culturels et de leurs substrats philologiques. Rien de plus fructueux, alors, que de se tourner vers le travail d'un historien chevronné comme Guy Stroumsa qui, à travers le concept de "religions abrahamiques », affronte le défi de l'analyse comparée du judaïsme rabbinique, du christianisme patristique et du premier islam, éclairant d'un jour nouveau l'effervescence religieuse de l'Antiquité tardive.
\end{abstract}

\section{Religious Science and the Challenge of the Comparative Approach : Critical Notes}

Several recent publications have offered French readers some practical tools for a global understanding of the monotheistic phenomenon. Two of them are examined in this article. It is clear that the major criterion of success in such matters is the comparative approach. The mere juxtaposition of informative notes is inadequate, but more ambitious syntheses can also fail because of an uneven knowledge of the different cultures and their philological bases. Nothing can be more useful, then, than the work of an experienced historian such as Guy Stroumsa. Using the concept of "Abrahamic religions", he truly confronts the challenges of comparative analysis and, through the study of rabbinic Judaism, patristic Christianity and the beginnings of Islam, sheds new light on the religious effervescence of Late Antiquity.

* À propos de : Dionigi Albera et Katell Berthelot (dir.), Dieu, une enquête. Judaïsme, christianisme, islam, ce qui les distingue, ce qui les rapproche, Paris, Flammarion, 2013, 1036 p.-[32 p. de pl.], ISBN : 978-2-08-121448-4. - Cyrille Michon et Denis Moreau (dir.), Dictionnaire des monothéismes, Paris, Seuil, 2013, 701 p., ISBN : 978-2-02-087147-1. - Guy G. Stroumsa, The Making of the Abrahamic Religions in Late Antiquity, Oxford, Oxford University Press, 2015 (« Oxford Studies in the Abrahamic Religions »), 225 p., ISBN : 978-0-19-873886-2. Les observations portant sur l'Enquête reviennent pour l'essentiel à Viviane Comerro de Prémare, celles relatives au Dictionnaire étant d'Alain Rauwel. 
Mener l'enquête sur Dieu : on conçoit qu'il n'ait pas fallu moins de mille pages pour une telle entreprise, qui décrit et compare les pratiques et les représentations religieuses des juifs, des chrétiens et des musulmans. Elle a été confiée à des historiens et à des anthropologues des religions, sous la direction d'une historienne du judaïsme antique et d'un ethnologue des religions monothéistes. Dans le même temps, un groupe de savants a eu l'idée d'offrir au grand public cultivé, sous un format commode, non un énième catalogue de notules factuelles sur les grandes figures et les hauts faits de l'histoire dite religieuse, mais un dictionnaire au sens noble, composé d'articles point trop contraints et consacrés à des concepts fondamentaux. Cela ne signifie d'ailleurs pas que les noms propres soient absents de la liste des entrées, mais le petit nombre de ceux qui ont été retenus, à l'exception d'un lieu (Jérusalem), correspond à ce que les auteurs désignent heureusement comme des «personnages conceptuels » : Abraham, Moïse, Jésus, Paul, Muhammad ne sont pas des acteurs éphémères apparus un moment sur le théâtre sacré, ils sont des fantômes qui hantent sans trêve les sanctuaires. Comme tels, ils sont aussi essentiels que les mots dieu, révélation, foi, dogme ou église à la compréhension de l'institutionnalisation des faits de croyance.

\section{LE COMPARATISME : NÉCESSITÉ ET APORIES}

Les deux volumes ont été confrontés d'emblée à la question capitale du comparatisme. Dieu : une enquête en adopte résolument la perspective, la revendique même, pour dépasser méthodologiquement l'étroitesse d'une démarche historiciste jugée trop contextualisante, mais aussi, sans le dire explicitement, l'exclusivisme théologique de chacun des monothéismes. Le principe retenu est donc le pari audacieux de confier à chaque auteur le traitement commun des trois religions, revenant à chacun de trouver le point d'équilibre entre continuité et rupture, ressemblances et spécificités. Dans le Dictionnaire des monothéismes, au contraire, les rédacteurs ont démissionné face au défi du comparatisme. "Judaïsme, christianisme, islam », lit-on en sous-titre. Et chaque article de présenter, quand le thème s'y prête, 
son paragraphe «judaïsme», son paragraphe « christianisme » et son paragraphe «islam »... Alors que les différences structurelles comme les points de convergence sautent aux yeux du lecteur, même non spécialiste, aucune présentation véritablement comparée n'a été tentée, nulle part. Il en allait pourtant de la qualification de l'ouvrage. Dumézil l'a dit, «le comparatisme est la méthode expérimentale des sciences humaines ». Une somme qui envisage des mondes et des milieux divers mais se contente d'en juxtaposer les analyses honore-t-elle pleinement le contrat de scientificité ? Que penser de trois articles «Bible» successifs ? Que penser, plus encore, d'un Dictionnaire des monothéismes qui écarte l'indispensable article «Monothéisme» et, à l'entrée «Unicité de Dieu», n'examine la question que pour l'islam?

Sur ce point, c'est le choix inverse qui a prévalu dans le chapitre de Dieu : une enquête écrit par Christian Boudignon et centré sur la difficulté de penser le Dieu un, qui constitue sans aucun doute le cœur de l'ouvrage autant par sa problématique que par la densité de sa réflexion à la fois historique et théologique. Le chapitre est divisé en deux parties : une longue histoire de l'idée monothéiste dont la source est située dans le judaïsme d'époque perse et hellénistique, puis une étude de sa «mécanique » traitant des rapports entre politique et monothéisme, de la logique de l'anathème et enfin de la nécessaire présence de figures intermédiaires entre le Dieu unique et l'homme, renvoyant à ce que Henry Corbin appelait le « paradoxe du monothéisme ».

S'adressant à un large public, les animateurs des deux entreprises ont dû affronter aussi la question du rapport entre discours assertif et discours critique. A priori, les choix de l'Enquête sont clairs: est affirmé, comme il se doit, le refus de tout essentialisme ; l'objet religion est envisagé dans sa diversité interne, dans ses transformations au cours de l'histoire, dans son extension géographique et culturelle. On évoque cependant, mais comme en passant, les «tendances lourdes » qui traversent les époques et les continents. L'expression, qui n'ose atteindre le seuil du concept, révèle une gêne à l'égard de la théologie dont les aperçus doctrinaux trouvent pourtant une large place dans l'ouvrage. Pourquoi éviter de dire qu'il y a des structures constantes dans l'expérience et dans l'interprétation et que ce sont elles qui fondent ce qu'on appelle une religion? Pourquoi ne pas reconnaître que l'on ne change pas de religion chaque fois que l'on 
change de siècle, de pays ou de milieu social ? Une gêne en partie comparable marque le Dictionnaire. Si ses directeurs se réclament d'une «perspective non apologétique », le lecteur n'est jamais vraiment au clair quant à la dimension confessante de l'entreprise. Le monde musulman est celui pour lequel la Wissenschaftlichkeit est la plus indiscutable. Les notices les plus importantes sont l'œuvre du regretté Alfred de Prémare, et elles comptent parmi les meilleures réussites de l'ensemble, offrant d'une recherche foisonnante, dont les occidentalistes sont moins familiers, des aperçus lumineux. Les rédacteurs voués au judaïsme ou au christianisme, en revanche, donnent parfois l'impression d'exposer les principes des systèmes qu'ils ont à analyser davantage selon un ordre catéchétique qu'en fonction d'un examen rationnel, ne s'astreignant pas toujours à la règle du « pas de côté » qui dénaturalise les discours reçus et les pratiques familières. Pour le monde chrétien, on appréciera cependant la présence de Job Getcha et de Vassa Kontouma qui, tout en faisant leur place aux réalités latines, rééquilibrent du côté de l'Orient une approche trop souvent exclusivement romaine. Cela étant, il est bien dommage que l'entrée « Orthodoxie » donne prétexte à une description de l'Église orthodoxe, ce qui relève du lieu commun, au lieu de s'engager dans une réflexion sur la place de la notion d'orthodoxie en christianisme qui eût été bien plus conforme au projet de l'ouvrage, tout comme «Réforme» est entendu comme Église réformée, non comme Ecclesia semper reformanda: concession à une approche de « culture générale » qui décevra les lecteurs un peu chevronnés.

\section{LES STRUCTURES ET L'HISTOIRE : UN DIALOGUE DÉLICAT}

Les présentations moins satisfaisantes pèchent souvent par défaut d'historicisation. On prendra l'exemple des notices «Mariage » et «Famille», car elles portent sur des objets que les formes modernes des grands monothéismes ont investis de valeurs étrangères aux élaborations initiales. Dans la première, on se contentera de sourire en voyant les «penseurs chrétiens » ramenés à l'improbable triade Chrysostome, Luther, Wojtyla! Dans la seconde, nonobstant de louables réticences au glissement du social au théologique, est esquissée malgré tout une «doctrine chrétienne de la famille » dont il eût fallu dire qu'elle n'a tout simplement pas existé avant le virage 
biopolitique de la papauté contemporaine. Et, quelques lignes plus haut, on ne lit pas avec moins de perplexité que « la famille est le lieu de l'avenir du peuple juif ». Où est, ici, l'apport scientifique ? Il semble bien que la question de la différence des sexes et de la reproduction biologique soit particulièrement difficile, car l'Enquête ne s'en sort pas mieux. Le chapitre VI, «La religion à l'épreuve du genre », laissait espérer une fructueuse intégration des questions les plus récentes; les auteurs ne donnent hélas qu'un catalogue de remarques très peu hiérarchisées où circoncision, excision, menstruation, homosexualité, voile... sont traités successivement sans que leur rapprochement produise vraiment de cohérence.

Le principe éditorial, a priori intéressant, de confier à un unique auteur le traitement des trois religions du Livre amène ainsi l'Enquête à flirter parfois avec l'imprécision, comme le montre le premier chapitre, centré sur la notion de Révélation. Gilles Dorival, fidèle à la représentation théologique, choisit de placer l'oralité à l'origine du parcours qu'il propose. Il examine ensuite la mise par écrit de la Parole et s'interroge sur la notion de canon ou plus largement de corpus. Un certain nombre des problèmes critiques que la philologie pose à la théologie sont soulevés : que faire des variantes du texte révélé ? Ou du texte révélé de l'autre ? Peut-on corriger le texte révélé ou le traduire ? La dernière partie du chapitre, consacrée à l'actualisation de la parole de Dieu, est intitulée « Retour de l'oralité », un titre qui se justifie pour traiter de la lecture liturgique et du sermon, moins ou pas du tout lorsque sont examinés les exigences de l'interprétation et les littéralismes contemporains. L'auteur remarque de façon fort pertinente à quel point «chez les anciens commentateurs juifs, chrétiens et musulmans, l'établissement du sens littéral nécessite un long et patient travail philologique, grammatical, sémantique, voire historique et anthropologique ». Mais quelques confusions apparaissent dans le traitement de l'islam. Ainsi la version définitive du Coran au $\mathrm{X}^{\mathrm{e}}$ siècle n'est-elle pas une hypothèse de la recherche (p. 40), mais l'amorce d'un processus de canonisation de ses différentes « lectures » autorisées. L'idée que l'on pourrait accroître le nombre des variantes non-canoniques par le dépouillement systématique des corans de toutes périodes (p. 50) doit être confrontée au fait que la tradition manuscrite reste pauvre jusqu'à nos jours et que, par ailleurs, des variantes non-canoniques ont été préservées par dizaines dans des ouvrages spécialisés de la tradition islamique. Du point de vue de 
l'auteur, l'islam se distinguerait du christianisme et du judaïsme sur un point fondamental : la sunna ne serait qu'une deuxième source de loi canonique et non une norme d'interprétation du texte révélé (p. 65). Il est pourtant avéré que la sunna ou tradition prophétique a constamment fourni au cours des siècles des clés d'interprétation orthodoxes à de grands commentateurs du Coran parmi lesquels Tabarî (m. 923), Baghawî (m. 1117) ou Ibn Kathîr (m. 1373) ; quant à Suyûtî (m. 1505), il a rédigé un commentaire tout entier spécialisé dans la relation entre le texte révélé et la tradition prophétique. D'autres points sont discutables. Sur quels fondements repose l'affirmation selon laquelle, dans les débuts de l'islam, l'idée que seul « le Coran explique le Coran » aurait été poussée à l'extrême (p. 70) ? L'auteur est-il assez familier du texte pour dire que « la thématique de la transmission comme épreuve serait absente du Coran» (p. 28) quand il suffit de regarder plusieurs passages et leurs commentaires pour affirmer le contraire $(Q 15,6 ; 37,36$ et passim, ou sur une autre épreuve liée à la proclamation orale $Q 20,114 ; 75,16)$ ? D'où vient l'idée que les musulmans auraient plus d'estime pour les juifs qui ont une loi, la Torah, que pour les chrétiens qui n'ont pas de loi dans l'Évangile (p. 51)?

Il faut souligner, en revanche, la réussite du pari éditorial dans l'étude magistrale d'Eric Chaumont intitulée «Qu'est-ce qu'obéir à la loi de Dieu », où le comparatisme tient pleinement ses promesses dans l'ordre de la réflexion. Les remarquables chapitres écrits par Dionigi Albera constituent, eux aussi, un point fort du volume. Ils traitent de l'inscription du croire dans le corps à travers les rites et le comportement humain culturellement réglé dans les préceptes de pureté touchant à l'alimentation, aux excrétions corporelles et à la sexualité. Le chapitre VII est sous-tendu par un problème de fond : comment concilier la transcendance absolue de Dieu et l'efficacité de la matérialité des rites ? Sont successivement évoqués les rites structurants du sacrifice, de la prière ${ }^{1}$, du jeûne et de l'ascèse ; puis l'institutionnalisation du croyant (selon l'acception de Bourdieu) par ces actes que sont la circoncision juive ou musulmane, le baptême chrétien (avec son statut particulier de sacrement), et

1. Mais la prière, ainsi que l'étude et la méditation qui lui sont associées, relève-t-elle vraiment et entièrement de la ritualité ? N'obscurcit-on pas la question en mêlant le cultuel et, pour le dire vite, le «spirituel »? 
le sacrifice animal dans l'islam; enfin dans une dernière partie, le retour du refoulé, qui désigne pour l'auteur «la contiguité avec le divin à travers la médiation d'objets sensibles » propres aux cosmothéismes de l'Antiquité : utilisation controversée des images ; investissement rituel des lieux naturels ; démarche votive qui repose sur un échange avec les puissances invisibles - tout cela dans une dynamique d'interdiction et de transformation, de répression ou d'acceptation résignée. L'auteur conclut sur la tension qui caractérise les trois religions monothéistes entre le souci de l'observance rituelle et son dépassement par une rationalisation éthique et une spiritualisation.

Le chapitre suivant (peut-être un peu long dans l'économie générale du volume) part du constat de l'abandon apparent de la pureté rituelle dans le christianisme, contrairement au judaïsme et à l'islam, pour rétablir une dynamique historique plus complexe. Sont évoquées les diverses théories tentant d'expliquer les interdits alimentaires bibliques, la dissolution de l'ensemble des interdits alimentaires dans l'Église d'Occident alors que ceux-ci restent présents en Orient, la parenté entre le décret apostolique et les interdits alimentaires du Coran, les tabous alimentaires concernant le porc dans les mondes hittites, sumériens et égyptiens dont vont hériter le judaïsme et l'islam. Une dernière partie concerne les ablutions et le contrôle de la sexualité. L'auteur en conclut que l'expérience de la pureté propre au monothéisme, qui implique une dissociation entre Dieu et le monde et un certain désenchantement de la nature et du monde animal, attribue à l'homme un rôle central dans le contrôle de son corps et l'éviction de la souillure.

Un peu plus loin, l'interrogation de Katell Berthelot sur « le rapport à autrui » part d'une problématique intéressante : définir « autrui » comme le membre de la communauté religieuse à laquelle on appartient, ou comme celui qui est à l'extérieur de cette communauté, voire comme tout être humain, change considérablement la donne. Dans chacune des divisions de son article, l'auteur ne manque pas de revenir sur la violence inhérente à toute appartenance communautaire. Ainsi dans sa première partie observe-t-elle que «la responsabilité vis-à-vis d'autrui » peut se transformer en coercition et en violence à son égard si l'intégrité de la communauté est en danger ; il y va alors de la responsabilité de chacun devant Dieu de la préserver. L'auteur note de même la distinction importante entre la bienveillance 
recommandée à l'égard de l'ennemi personnel et la détestation de l'ennemi de Dieu (impie, hérétique, apostat, etc.) qui doit être, lui, combattu. Enfin, dans la dernière partie, les conséquences de l'exclusivisme religieux sont évidemment rappelées ainsi que ses manifestations connues contre la déviance ou l'hétérodoxie. Il reste cependant que le ton général de l'article est irénique. Celui-ci s'achève sur l'émergence d'une nouvelle forme d'éthique religieuse qui déborderait les frontières de la communauté d'appartenance pour rejoindre l'humanité entière "à condition de dépasser le littéralisme, le fondamentalisme et l'intégrisme ». Dépasser ? On peut s'interroger sur la mise en ouvre pratique de cette dialectique. Le tempo de l'article s'inscrit dans le rythme binaire d'une thèse (les aspects positifs de l'appartenance communautaire) et d'une antithèse (les aspects négatifs) dont on attendrait le dépassement dialectique dans un "élargissement nouveau de l'éthique de responsabilité » posé en conclusion. Il semble pourtant que la logique de la violence religieuse ne peut être conjurée ni par une pirouette en forme de citation (un hadith dit que... Philon dit que...), ni par un souhait ou même une espérance. Comme le reconnaît l'auteur elle-même, c'est la philosophie des Lumières dont est issue l'idéologie universaliste des droits de l'homme qui a influencé les religions monothéistes pour les conduire à une condamnation de l'esclavage. La confrontation mériterait d'être élargie à d'autres questions. Cette « responsabilité à l'échelle de l'humanité tout entière » ne vient-elle pas de la philosophie plutôt que de la religion ? Ou à l'intérieur de la religion, de la mystique plutôt que de l'institution communautaire ? On touche là au socle dur de la religion : celui-ci est le bien de tous, non celui d'une élite savante. La question est de savoir si l'homme ordinaire est fait pour absorber l'universel.

Le fil rouge de la violence religieuse court ainsi, au moins implicitement, à travers toute l' Enquête. La perspective adoptée étant historique et anthropologique - et non théologique (vrai/faux) - les «préjugés » et «stéréotypes » que les directeurs dénoncent dans leur introduction comme obstacles à la recherche auraient pu et sans doute dû être pris en compte en tant que représentations et sources constantes de tensions et de violence. Une violence que Jan Assmann décrit comme inhérente au monothéisme de l'alliance et de la fidélité qui sépare les hommes entre amis et ennemis. L'ouvrage paie ici le prix de ses choix : s'il repose sur un savoir 
universitaire solidement constitué autant en matière d'histoire et de théologie que d'anthropologie, s'il tombe rarement dans un catalogue des ressemblances et des différences qui aurait pu être le fruit de la perspective adoptée, la distanciation des auteurs ne s'autorise pas toujours à nous donner des clés pour comprendre la violence inhérente à une certaine appartenance religieuse aussi bien dans le passé que dans le monde contemporain.

Le Dictionnaire des monothéismes ouvre lui aussi une vaste carrière de matériaux, dont beaucoup sont solides. Mais le lecteur est laissé dans l'obligation de construire lui-même l'édifice dont les blocs ont été soigneusement taillés. Qu'on en juge d'après un cas : à l'article «Rabbin », l'idée de Yeridat ha-Dorot évoque inévitablement la notion chrétienne de tradition. Mais à l'entrée «Tradition» l'optique juive est différente, et il n'y a pas de partie chrétienne, qu'il faut aller chercher sous «Révélation »! Comment, dans ces conditions, vérifier la validité d'une intuition ? De même, l'excellent article «Islam » note l'évolution du concept vers un sens géographique, le dar al-Islam. Il y a donc exactement le même type de territorialisation que pour christianitas. Mais il n'y a pas d'article «Chrétienté », l'entrée «Christianisme » ne porte que sur le regard musulman, et «Chrétiens » n'est gratifié que d'une trentaine de lignes insuffisantes à tous points de vue... Les difficultés soulignées ici sont peut-être simplement celles dont les auteurs sont bien conscients lorsqu'ils terminent l'article «Théologie » par ce juste constat : le débat qui opposa longtemps théologie et philosophie a changé de nature, et ce sont désormais les sciences religieuses qui demandent aux théologies de se dire selon l'ordre des raisons.

\section{UN ESSAI AMBITIEUX}

Bien différent est le projet littéraire de Guy Stroumsa dans son Making of the Abrahamic religions: il s'agit ici d'un véritable essai, qui vise plus à rapprocher des données éparses comme on frappe deux silex pour en faire jaillir la flamme qu'à donner un panorama encyclopédique d'un monde géographiquement et chronologiquement fort étendu, soit le Proche et Moyen-Orient tardo-antique, du triomphe du christianisme à l'expansion de l'islam, 
selon une chronologie à laquelle les travaux de l'école historique anglo-saxonne nous ont familiarisés. L'origine des chapitres va d'ailleurs en ce sens, puisqu'ils reprennent dans une perspective nouvelle des textes précédemment publiés dans divers recueils, parfois dirigés par l'auteur lui-même. Fidèle à une méthode déjà brillamment illustrée, G. Stroumsa ne considère jamais tel ou tel groupe religieux isolément, selon une approche monographique- sauf à attirer l'attention sur telle communauté marginalisée par l'histoire. Il concentre plutôt son intérêt sur les relations qui, inévitablement, se nouent en tous sens dans le religious commonwealth oriental. Si la situation initiale amène à prendre acte d'une « révolution religieuse de l'Antiquité tardive ", commune en bien des aspects à toutes les traditions du monde méditerranéen et dont l'auteur rassemble les principaux traits autour de la formule désormais classique de « fin du sacrifice ${ }^{2}$ », le tableau du VII ${ }^{\mathrm{e}}$ siècle est bien différent: le christianisme, secte interdite puis acceptée par Rome, est redevenu en Méditerranée orientale une secte tolérée par les califes, désormais à jeu égal avec le judaïsme dans la condition de dhimmi. « Surprenante continuité de la forme-secte !»

Dans ce cadre, G. Stroumsa souligne la force de plusieurs modèles agrégateurs, à commencer par le prophétisme. De manière très efficace, il l'aborde par la question récurrente des « faux prophètes ». L'âge des prophéties serait-il clos ? Il est de l'intérêt des institutions de le soutenir. Mais de nombreux « charismatiques ambulants » (Gerd Theissen) entendent bien, génération après génération, démentir ce verdict. Leur influence ne dessine pas une courbe décroissante, au contraire : le VII $^{\mathrm{e}}$ siècle connaît une «nouvelle vague d'attente eschatologique », dans laquelle s'inscrit pleinement la révélation de Mahomet. Du point de vue de l'islam, bien que le texte coranique soit peu explicite à ce sujet, il vient sceller les prophéties. De celui des autres monothéismes, il est le faux prophète par excellence, selon une typologie qui puise aussi bien à l'Ancien qu'au Nouveau Testament : histoire connectée des prophétismes, pourrait-on dire.

Une autre structure se renforce entre $\mathrm{IV}^{\mathrm{e}}$ et $\mathrm{VII}^{\mathrm{e}}$ siècle : c'est celle de la communauté - qu'elle se présente comme force d'affirmation ou cadre de repli et de résistance. L'auteur suggère ainsi qu'amenés à

2. Guy G. Stroumsa, La fin du sacrifice : les mutations religieuses de l'Antiquité tardive, Paris, Odile Jacob, 2005. 
faire front par leur marginalisation même, les juifs du Proche-Orient ancien en ont retiré une identité collective plus solide, après le flottement qui avait suivi la destruction du Temple. Et la tolérance de l'islam envers les chrétiens au nom de la Loi écrite est-elle d'une autre nature que celle qu'avaient cultivée ces mêmes chrétiens face aux juifs en considération de l'ancienne alliance ? On voit que la dimension politique est ici centrale. Dans son important chapitre VII, G. Stroumsa se livre à une brillante enquête autour de la notion de théocratie, forgée par Josèphe pour rendre compte de l'ordre entièrement nouveau instauré par Moïse le législateur. Or c'est aussi autour de la figure de Moïse que s'articule la démonstration mimétique d'Eusèbe dans sa Vie de Constantin: l'empereur est nouveau Moïse plutôt que nouveau David dans la mesure où il est avant tout nomothetes. Rebondissant sur une remarque de Polymnia Athanassiadi, Stroumsa défend alors l'idée d'une certaine similitude entre modèle eusèbien et système califal. Plus que du Prophète, c'est de Dieu lui-même que le calife est représentant et vicaire. Par-delà les différences considérables entre milieux se laissent donc discerner des lignes de force qui unissent l'univers de pensée hébraïque au premier islam, par le biais des élaborations théologico-politiques de l'âge constantinien.

Comment en serait-il autrement quand l'arrière-plan culturel des contacts religieux est largement commun, fondé sur une tradition herméneutique partagée, au moins entre juifs et chrétiens ? Par-delà la question des langues, G. Stroumsa envisage la possibilité de typologies textuelles croisées. Le Midrash et les catenae scripturaires, dont le développement est à peu près concomitant, lui semblent mériter d'être rapprochés, comme l'avait proposé Nicholas de Lange, tout comme, peut-être, les formes de poésie synagogale et les kontakia byzantins. Et au terme du parcours, ne faut-il pas envisager l'islam primitif comme fruit d'une séculaire preparatio coranica, à laquelle ont collaboré à leur insu les Pères de l'Église et les rabbins à travers le renouveau éthique du «moment abrahamique »? G. Stroumsa en est convaincu. Rassemblant les nombreuses observations relatives aux similitudes entre prédication du Prophète et substrat biblique, il souligne que le monde arabique était beaucoup moins marginal qu'on ne l'a dit parfois, que des « flux d'idées » circulaient abondamment entre groupes et communautés, y compris sous des formes plus ou moins mixtes, de type judéo-chrétien, qui ont pu longtemps survivre 
dans ces régions. Sans nécessairement aller jusqu'à voir dans l'islam, avec Theodor Nöldeke, une «forme arabe du christianisme», il convient d'en interpréter l'émergence à l'aune de rapports répétés qui prennent tout leur sens lorsque, comme G. Stroumsa, on les inscrit dans une polyphonie religieuse de longue durée. Jean Damascène, un personnage particulièrement important de ce grand récit, n'hésitait pas, tout hostile qu'il fût, à considérer la nouvelle religion d'Arabie comme une hérésie, non comme un produit idéologique extérieur à son système dogmatique.

Le paradigme vetus Israel/verus Israel apparaît particulièrement apte à exprimer le noyau unitaire du système. La polémique chrétienne s'est nourrie de l'argument selon lequel, la synagogue étant aveugle invétérée, c'est l'Église qui est le nouveau peuple élu, avant que Mahomet et ses disciples ne stigmatisent comme déviants et juifs et chrétiens, se proclamant, eux, vrais fils d'Abraham. Abraham : tel est bien le point de référence. La vraie koinè, selon G. Stroumsa, est la filiation abrahamique, et il invite dans sa conclusion à considérer judaïsme rabbinique, christianisme patristique et islam primitif comme autant de visages ou, mieux, d' « hérésies », en un sens purement descriptif, de l'unique mouvement abrahamique. On pourra penser qu'il y a une bonne pointe d'utopie dans ce grand élan œcuménique, bien dans la ligne de la chaire qu'occupa l'auteur à Oxford, sous le vocable de "Religions abrahamiques ${ }^{3}$ », et de la collection qui en est issue. Abraham est aussi, il faut l'avouer, une figure beaucoup plus consensuelle que Moïse, surinvesti par la tradition occidentale en toutes ses dimensions ${ }^{4}$.

Reste que la vraie question à soulever, au-delà des querelles de mots, est celle de la force heuristique du modèle construit par G. Stroumsa. Incontestablement, elle est grande. C'est par le recours à des propositions englobantes, avec tout ce que cela suppose d'audace, que l'on peut dépasser la vanité des monographies juxtaposées. En choisissant d'ouvrir une percée à travers une matière immense selon un axe dont on est bien conscient qu'il n'est jamais le seul possible, on se donne les moyens de faire

3. Paraît en même temps que l'essai de G. Stroumsa un ouvrage de synthèse qu'il co-dirige avec A. Silverstein, The Oxford handbook of the Abrahamic religions.

4. Ne rappelons ici que Bruno Karsenti, Moïse et l'idée de peuple, Paris, Cerf, 2012. 
surgir un peu d'intelligibilité par-delà le foisonnement des faits - sans d'ailleurs que cette riche et savoureuse diversité soit jamais amoindrie ou nivelée. À suivre sur cette route de crête un maître comme Guy Stroumsa, le lecteur recueillera un trésor d'intuitions et d'idées inconnu aux manuels ad usum delphini, fussent-ils les mieux pensés.

vivianedepremare@orange.fr a.rauwel@wanadoo.fr 